\title{
Respostas fisiológicas da mandioca à aplicação de herbicidas
}

\section{Cassava physiological responses to the application of herbicides}

\author{
Evander Alves Ferreira ${ }^{1}$; Christiano da Conceição de Matos $^{2}$; \\ Edimilson Alves Barbosa²; Daniel Valadão Silva ${ }^{3 *}$; \\ José Barbosa dos Santos ${ }^{4}$; Gustavo Antonio Mendes Pereira ${ }^{2}$; \\ Autieres Teixeira Faria ${ }^{2}$; Cícero Teixeira da Silva ${ }^{5}$
}

\section{Resumo}

A análise da fluorescência da clorofila $a$ vem sendo utilizada para melhorar o entendimento dos mecanismos da fotossíntese, bem como, na avaliação da capacidade fotossintética das plantas alterada por estresses bióticos ou abióticos. Objetivou-se com esse trabalho avaliar a sensibilidade de plantas de mandioca à herbicidas com diferentes mecanismos de ação, bem como, os danos causados pela aplicação desses herbicidas no aparato fotossintético destas plantas. Para isso foi realizado um experimento no delineamento em blocos casualizados, com quatro repetições. Os tratamentos foram constituídos da aplicação em pós-emergência da mandioca dos seguintes herbicidas: bentazon, clomazone, fomesafen, fluazifop-p-buthyl, glyphosate, nicosulfuron, chlorimuron, fluazifop-p-buthyl + fomesafen, sulfentrazone, além de uma testemunha sem aplicação. As avaliações de intoxicação visual e fluorescência da clorofila $a$ foram realizadas aos 2, 9, 16 e 23 dias após a aplicação dos herbicidas. Os herbicidas avaliados afetaram de forma diferenciada as plantas de mandioca, sendo que, o glyphosate e sulfentrazone promoveram a morte das plantas. Os herbicidas clomazone, fomesafen, fluazifop-pbuthyl e chlorimuron-ehtyl causaram baixa intoxicação as plantas de mandioca e não afetaram a relação Fv/Fm e a ETR. No entanto, para o nicosulfuron e a mistura fluazifop-p-buthyl + fomesafen os valores da Fv/Fm estiveram abaixo do ideal nas primeiras épocas de avaliação mas as plantas tratadas com esses herbicidas se recuperam. As variáveis fisiológicas avaliadas podem ser utilizadas como forma de avaliar a seletividade de herbicidas a mandioca, pois apresentaram respostas semelhantes aos observados para a intoxicação visual da cultura.

Palavras-chave: Manihot esculenta Crantz, fotossíntese, taxa de transporte de elétrons, fluorescência da clorofila $a$

\begin{abstract}
Analysis of chlorophyll $a$ fluorescence has been used to improve the understanding of the mechanisms of photosynthesis, as well as in the evaluation of plant photosynthetic capacity altered by biotic or abiotic stresses. The objective of this study was to evaluate the sensitivity of cassava plants to herbicides with different mechanisms of action, as well as the damage caused by the application of herbicides on the photosynthetic apparatus of these plants. An experiment was conducted in a randomized block design with four replications. The treatments were constituted of the application of the following post-
\end{abstract}

${ }^{1}$ Dr. em Fitotecnia, Eng ${ }^{\circ}$ Agr $^{\circ}$, UFVJM, Universidade Federal dos Vales do Jequitinhonha e Mucuri, Diamantina, MG. E-mail: evanderalves@gmail.com

2 Discentes do Curso de Doutorado em Fitotecnia, UFV, Universidade Federal de Viçosa, Viçosa, MG. E-mail: chrisconmatos@ yahoo.com.br; agroedi1000@yahoo.com.br; gustavogamp@hotmail.com; autieresteixeira@yahoo.com.br

3 Dr. em Fitotecnia, Eng ${ }^{\circ}$ Agr $^{\circ}$, UFV, Viçosa, MG. E-mail: danielvaladaos@yahoo.com.br

4 Dr. em Fitotecnia, Eng ${ }^{\mathrm{o}}$ Agr $^{\circ}$, UFVJM, Diamantina, MG. E-mail: jbarbosasantos@yahoo.com.br

5 Discente do Curso de Mestrado em Produção Vegetal, UFVJM, Diamantina, MG. E-mail: ciceroagronomia@hotmail.com

* Autor para correspondência 
emergence herbicides in cassava: bentazon, clomazone, fomesafen, fluazifop-p-buthyl, glyphosate, nicosulfuron, chlorimuron, fluazifop-p-buthyl + fomesafen, sulfentrazone, besides a control without application. The visual intoxication and chlorophyll $a$ fluorescence assessments were performed at 2, 9, 16 and 23 days after herbicide application. The herbicides evaluated affected differently the cassava plants. Sulfentrazone and glyphosate promoted plant death. Herbicides clomazone, fomesafen, fluazifop-p-buthyl and chlorimuron-ehtyl caused low toxicity to cassava plants and did not affect the ratio Fv / Fm and ETR. However, for the mixture nicossulfuron and fluazifop-p-buthyl + fomesafen values of $\mathrm{Fv} / \mathrm{Fm}$ were suboptimal in the first evaluation times but plants treated with these herbicides had recovered. Physiological evaluations can be used as a way to evaluate the selectivity of herbicides in cassava crop as presented similar answers to those observed for visual intoxication symptoms.

Key words: Manihot esculenta Crantz, photosynthesis, electron transport rate, chlorophyll a fluorescence

\section{Introdução}

A mandioca (Manihot esculenta Crantz) é uma espécie amplamente cultivada em todo território brasileiro, sendo considerado alimento básico para mais de 700 milhões de pessoas em pelo menos 105 países. Recentemente, têm sido realizados vários estudos visando melhorar o manejo e eficiência produtiva da cultura. Dentre os gargalos ainda existentes destacam-se as poucas informações sobre controle químico de plantas daninhas (SILVA et al., 2012a).

Em estudos de seletividade de herbicidas é importante avaliar as intoxicações provocadas por eles, bem como os efeitos sobre o crescimento e a produtividade da planta cultivada (NEGRISOLI et al., 2004; GALON et al., 2009). No entanto, muitas vezes os danos causados pelos herbicidas não são visíveis e o tempo de avaliação não é suficiente para que se constate intoxicação da cultura pelo produto aplicado. Deste modo, alguns autores têm proposto avaliar as respostas fisiológicas das culturas como forma de selecionar herbicidas com potencial para aplicação (GALON et al., 2010; SILVEIRA et al., 2012, 2013; TORRES et al., 2012).

Para analisar a alteração na capacidade fotossintética causada pelo uso de herbicidas, são utilizados equipamentos que fazem mensurações que variam desde a rápida identificação de injúrias causadas ao aparato fotossintético, mesmo com sintomas não visíveis, até análises que envolvem, principalmente, a fluorescência da clorofila $a$, trocas gasosas nas plantas, teor de clorofila e taxa de transporte de elétrons (GIROTTO et al., 2010).
O uso de parâmetros de fluorescência tem sido difundido principalmente no estudo de fotossíntese por ser um método que, além de não destrutivo, permite analisar qualitativa e quantitativamente a absorção e o aproveitamento da energia luminosa através do fotossistema II e as possíveis relações com a capacidade fotossintética (TORRES NETTO et al., 2005; FERRAZ et al., 2014). Considerando o processo fotossintético, a luz é absorvida por pigmentos do complexo-antena, que, excitados, transferem energia para os centros de reação dos fotossistemas I e II ( $\mathrm{P}_{700}$ e $\mathrm{P}_{680}$, respectivamente) (YOUNG; FRANK, 1996). Quando ocorre excesso de energia, esta pode ser dissipada na forma de fluorescência (KRAUSE; WINTER, 1996). Assim, um dos métodos de monitoramento da inibição ou redução na transferência de elétrons entre os fotossistemas da planta sob estresse, que pode ser observada ainda em folhas intactas, é a fluorescência da clorofila (MAXWELL; JOHNSON, 2000), em que a redução na dissipação da energia pelo processo fotoquímico é refletida por incremento correspondente na fluorescência.

A análise da fluorescência da clorofila $a$ vem sendo utilizada para melhorar o entendimento dos mecanismos da fotossíntese e na avaliação da capacidade fotossintética alterada por estresses bióticos ou abióticos pelos quais as plantas possam sofrer, como temperatura (OLIVEIRA; ALVES; MAGALHÃES, 2002), radiação (MAZZA et al., 2000), deficiência hídrica (RODEN; BALL, 1996), salinidade (BELKHODJA et al., 1994), presença de insetos (BOWN; HALL; MACGREGOR, 2002), herbicidas (IRELAND; PERCIVAL; BAKER, 
1986; CATUNDA et al., 2005) etc. Para esse tipo de avaliação são utilizados fluorômetros de luz modulada.

Objetivou-se neste trabalho avaliar a sensibilidade de plantas de mandioca à herbicidas com diferentes mecanismos de ação, bem como, os danos causados pela aplicação desses herbicidas no aparato fotossintético das plantas.

\section{Material e Métodos}

O experimento foi realizado em ambiente protegido, no Departamento de Agronomia da Universidade Federal dos Vales do Jequitinhonha e
Mucuri (UFVJM), Diamantina-MG, no ano de 2012. Foram utilizadas amostras de Argissolo VermelhoAmarelo, textura argilosa, que após secagem ao ar foram peneirados em malha de $5 \mathrm{~mm}$. A análise química e granulométrica do solo encontra-se na Tabela 1. Para adequação do substrato quanto à nutrição foram aplicados o equivalente a, $220 \mathrm{~kg}$ $\mathrm{ha}^{-1}$ de superfosfato simples e $40 \mathrm{~kg} \mathrm{ha}^{-1}$ de cloreto de potássio. A adubação nitrogenada foi realizada em cobertura aos 30 dias após a emergência da cultura, na dose de $40 \mathrm{~kg} \mathrm{ha}^{-1}$ de ureia previamente dissolvida em água. As unidades experimentais constaram do plantio de manivas do cultivar IAC12 em vasos plásticos com volume de $8 \mathrm{dm}^{-3}$.

Tabela 1. Resultado da análise física e química de um Argissolo Vermelho-Amarelo.

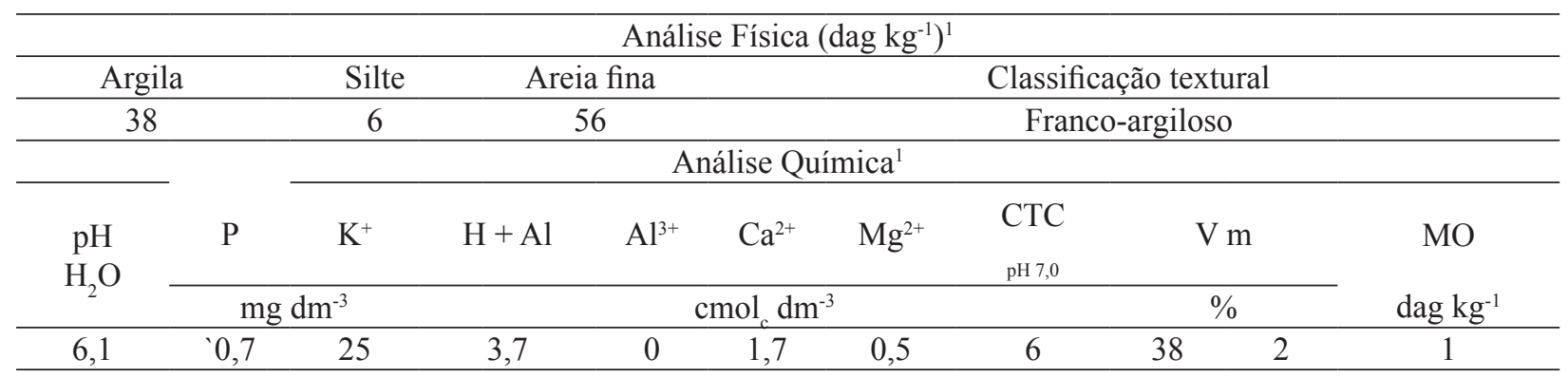

${ }^{1}$ Análises realizadas no Laboratório de Análise de Solos da Universidade Federal dos Vales do Jequitinhonha e Mucuri, DiamantinaMG.

Fonte: Elaboração dos autores.

O delineamento experimental utilizado foi em blocos casualizados, com quatro repetições. Os tratamentos foram constituídos da aplicação dos seguintes herbicidas: bentazon (720 i. a. g ha-1), clomazone (340 i. a. g ha-1), fomesafen (250 i. a. $\mathrm{g}$ ha $\left.^{-1}\right)$, fluazifop-p-buthyl (250 i. a. g ha $\left.{ }^{-1}\right)$, glyphosate (360 i. a. g ha-1), nicosulfuron (60 i. a. $g h^{-1}$ ), chlorimuron-ehtyl (15 i. a. g ha-1 ${ }^{-1}$, fluazifop-p-buthyl + fomesafen $\left(200+250\right.$ i. a. $\left.\mathrm{g} \mathrm{ha}^{-1}\right)$ e sulfentrazone (600 i. a. $\mathrm{g} \mathrm{ha}^{-1}$ ), além de uma testemunha sem aplicação.

A aplicação dos herbicidas foi realizada aos 45 dias após o plantio da mandioca (DAP), quando as plantas apresentavam cerca de $20 \mathrm{~cm}$ de altura e aproximadamente 15 folhas completamente expandidas. Para a aplicação utilizou-se de um pulverizador costal pressurizado à $\mathrm{CO}_{2}$, com pressão constante $200 \mathrm{kPa}$, equipado com uma barra de dois bicos de indução de ar TTI 11002, trabalhando a uma altura de $50 \mathrm{~cm}$ do alvo, com velocidade de 1 $\mathrm{ms}^{-1}$, atingindo faixa aplicada de $100 \mathrm{~cm}$ de largura, propiciando volume de calda de $200 \mathrm{~L} \mathrm{ha}^{-1}$.

Aos 2, 9, 16 e 23 DAA dos herbicidas realizou-se a análise da eficiência fotoquímica do fotossistema II das folhas das plantas de mandioca com o uso de um fluorômetro, sendo as pinças do aparelho colocadas no terço médio da primeira folha completamente expandida das plantas de mandioca. As medições 
foram feitas após 30 minutos de adaptação ao escuro, no período noturno, com emissão de um pulso de luz saturante de $0,3 \mathrm{~s}$, sob frequência de $0,6 \mathrm{KHz}$, quando se avaliou também a fluorescência inicial $\left(\mathrm{F}_{\mathrm{o}}\right)$, fluorescência máxima $\left(\mathrm{F}_{\mathrm{m}}\right)$, a razão entre a fluorescência variável e fluorescência máxima $\left(\mathrm{F}_{\mathrm{v}} /\right.$ $\mathrm{F}_{\mathrm{m}}$ ) e a taxa de transporte de elétrons (ETR $-\mu \mathrm{Mols}$ elétrons m-2 s-1).

Nestas mesmas datas (2, 9, 16 e 23 DAA) avaliou-se também a intoxicação visual da cultura. Os sintomas foram avaliados com uso de escala percentual de notas variando entre 0 (zero) e 100 (cem), onde 0 implica ausência de quaisquer injúrias e 100, a morte da planta.

Os dados referentes à relação $\mathrm{Fv} / \mathrm{Fm}$ foram submetidos à análise de variância e apresentados na forma de gráficos para cada herbicida com o desvio padrão nas diferentes épocas de avaliação. Os dados referentes à Fo, Fm, relação Fv/Fm, ETR e intoxicação visual foram submetidos a análise de variância e as médias comparadas pelo teste de Scott Knott a 5\% de probabilidade.

\section{Resultados e Discussão}

Os herbicidas sulfentrazone e glyphosate promoveram as maiores intoxicações às plantas de mandioca independente da época de avaliação (Tabela 2). Aos 9 DAA constatou-se a formação de quatro grupos de acordo com o grau de intoxicação, sendo que o glyphosate e o sulfentrazone, provocaram intoxicação nas plantas de mandioca superiores a $90 \%$, e o bentazon, o clomazone, o fomesafen e o fluazifop-p-buthyl os valores da variável foi próximo a $20 \%$.

Tabela 2. Intoxicação de mandioca (\%), avaliada visualmente, em diferentes épocas após a aplicação de herbicidas.

\begin{tabular}{lrrcc}
\hline Tratamentos & 2 DAA* & 9 DAA & 16 DAA & 23 DAA \\
Bentazon & $5,00 \mathrm{~b}$ & $21,25 \mathrm{~b}$ & $6,25 \mathrm{~b}$ & $6,00 \mathrm{c}$ \\
Clomazone & $6,75 \mathrm{~b}$ & $18,75 \mathrm{~b}$ & $7,50 \mathrm{~b}$ & $3,75 \mathrm{c}$ \\
Fomesafen & $7,50 \mathrm{~b}$ & $20,00 \mathrm{~b}$ & $5,00 \mathrm{~b}$ & $4,70 \mathrm{c}$ \\
Fluazifop-buthyl & $5,00 \mathrm{~b}$ & $8,75 \mathrm{c}$ & $1,25 \mathrm{c}$ & $1,25 \mathrm{c}$ \\
Glyphosate & $5,00 \mathrm{~b}$ & $91,25 \mathrm{a}$ & $100,00 \mathrm{a}$ & $100,00 \mathrm{a}$ \\
Nicossulfuron & $10,50 \mathrm{~b}$ & $11,75 \mathrm{c}$ & $8,25 \mathrm{~b}$ & $5,00 \mathrm{c}$ \\
Chlorimuron-ethyl & $6,75 \mathrm{~b}$ & $15,00 \mathrm{c}$ & $11,25 \mathrm{~b}$ & $13,75 \mathrm{~b}$ \\
Fomesafen+Fluazifop & $12,50 \mathrm{~b}$ & $21,25 \mathrm{~b}$ & $19,25 \mathrm{~b}$ & $18,75 \mathrm{~b}$ \\
Sulfentrazone & $23,75 \mathrm{a}$ & $91,00 \mathrm{a}$ & $100,00 \mathrm{a}$ & $100.00 \mathrm{a}$ \\
Testemunha & $0,00 \mathrm{~b}$ & $0,00 \mathrm{~d}$ & $0,00 \mathrm{c}$ & $0,00 \mathrm{c}$ \\
\hline CV $(\%)$ & \multicolumn{3}{c}{} \\
\hline
\end{tabular}

*Escala de notas em que a nota $0 \%$ significa em ausência de quaisquer injúrias e $100 \%$ a morte da mandioca.

Médias seguidas pela mesma letra na coluna não diferem entre si pelo teste de Scott Knott a 5\% de probabilidade. DAA: Dias após a aplicação.

Fonte: Elaboração dos autores.

Comportamento semelhante foi observado aos 16 e 23 DAA, entretanto, o nível de intoxicação reduziu para valores inferiores a $10 \%$ nas plantas tratadas com bentazon, clomazone, fomesafen, fluazifop-p-buthyl (Tabela 2). Observou-se também a morte das plantas de mandioca tratadas com glyphosate e sulfentrazone. Silva et al. (2012b) constaram que a aplicação em pós-emergência do bentazon causou valores de intoxicação semelhantes ao observados neste trabalho. Entretanto, o mesmo herbicida não foi capaz de reduzir o acúmulo de biomassa das plantas de mandioca. Naquele trabalho o sulfentrazone, apesar de não promover a morte das plantas de mandioca, foi capaz de causar altos valores de intoxicação e redução no crescimento das plantas. 
Ao avaliar a florescência inicial da clorofila (Fo) de plantas de mandioca aos 2 DAA, constatou-se a formação de três grupos de herbicidas, sendo que, a testemunha livre da aplicação do produto encontrou-se no grupo intermediário juntamente com os herbicidas clomazone, fluazifop-p-buthyl, chlorimuron-ehtyl e sulfentrazone (Tabela 3). Para o bentazon observou-se maior valor relacionado à Fo e pode ser indicativo de danos estruturais nos centros de reação do Fotossistema II ou comprometimento no transporte de energia de excitação dos complexos antena para os centros de reação (BOLHÀR-
NORDENKAMPF et al., 1989). Segundo os mesmos autores a Fo nem sempre é uma constante, o seu valor pode aumentar caso os centros de reação do PSII estejam comprometidos, ou se a transferência da energia de excitação da antena para os centros de reação esteja prejudicada. Nas demais avaliações (9 e 16 DAA) verificaram-se que a aplicação dos herbicidas glyphosate e sulfentrazone causaram sintomas irreversíveis de intoxicação das plantas de mandioca que provocaram a morte da mandioca a partir dos 16 DAA (Tabela 3). Já aos 23 DAA observou-se que as somente o bentazon, glyphosate e sulfentrazone diferiram-se da testemunha.

Tabela 3. Fo - Fluorescência inicial da clorofila $a$ (elétrons quantum ${ }^{-1}$ ) de plantas de mandioca em diferentes épocas após a aplicação dos herbicidas.

\begin{tabular}{lcccc}
\hline Tratamentos & 2 DAA & 9 DAA & 16 DAA & 23 DAA \\
Bentazon & $257,00 \mathrm{a}$ & $190,00 \mathrm{a}$ & $167,25 \mathrm{~b}$ & $125,00 \mathrm{a}$ \\
Clomazone & $190,25 \mathrm{~b}$ & $156,25 \mathrm{a}$ & $195,75 \mathrm{a}$ & $82,00 \mathrm{~b}$ \\
Fomesafen & $134,25 \mathrm{c}$ & $147,25 \mathrm{a}$ & $175,55 \mathrm{~b}$ & $63,25 \mathrm{~b}$ \\
Fluazifop-buthyl & $137,00 \mathrm{c}$ & $148,50 \mathrm{a}$ & $146,50 \mathrm{~b}$ & $61,50 \mathrm{~b}$ \\
Glyphosate & $157,50 \mathrm{~b}$ & $0,00 \mathrm{~b}$ & $0,00 \mathrm{c}$ & $0,00 \mathrm{c}$ \\
Nicosulfuron & $160,75 \mathrm{~b}$ & $165,50 \mathrm{a}$ & $199,50 \mathrm{a}$ & $74,25 \mathrm{~b}$ \\
Chlorimuron-ethyl & $132,00 \mathrm{c}$ & $142,25 \mathrm{a}$ & $178,00 \mathrm{a}$ & $72,25 \mathrm{~b}$ \\
Fomesafen+Fluazifop & $156,75 \mathrm{~b}$ & $148,75 \mathrm{a}$ & $159,00 \mathrm{~b}$ & $70,00 \mathrm{~b}$ \\
Sulfentrazone & $125,25 \mathrm{c}$ & $0,00 \mathrm{~b}$ & $0,00 \mathrm{c}$ & $0,00 \mathrm{c}$ \\
Testemunh & $166,25 \mathrm{~b}$ & $138,25 \mathrm{a}$ & $196,00 \mathrm{a}$ & $67,75 \mathrm{~b}$ \\
\hline CV $(\%)$ & \multicolumn{3}{c}{} \\
\hline
\end{tabular}

Médias seguidas pela mesma letra na coluna não diferem entre si pelo teste de Scott Knott a 5\% de probabilidade. DAA: Dias após a aplicação.

Fonte: Elaboração dos autores.

O valor de Fo é alterado por estresses do ambiente que causam alterações estruturais nos pigmentos fotossintéticos do PSII. Estresse por temperaturas infraótimas decresce significativamente os valores de Fo e o estresse por temperaturas supra-ótimas é caracterizado por incrementar drasticamente os valores de Fo. Os valores elevados desta variável após a aplicação do bentazon estão de acordo com o observado por outros autores em aplicações de herbicidas inibidores do fotossistema II (IRELAND; PERCIVAL; BAKER, 1986; CATUNDA et al., 2005).

Observou-se aos 2 DAA a formação de quatro grupos de herbicidas quanto a fluorescência máxima da clorofila $(\mathrm{Fm})$, sendo que, a testemunha juntamente com a maior parte dos produtos se alocaram no grupo com os maiores valores de Fm, já as plantas tratadas com glyphosate, fluazifopp-buthyl + fomesafen, sufentrazone e bentazon mostraram redução nos valores de Fm em relação a testemunha para essa época de avaliação (Tabela 4). $\mathrm{Na}$ avaliação realizada aos $23 \mathrm{DAA}$, constatou-se a formação de apenas dois grupos de herbicidas em relação aos valores de Fm, sendo que plantas tratadas com glyphosate e sulfentrazone se enquadraram no grupo de herbicidas que provocaram a morte das plantas de mandioca (Tabela 3). 
Tabela 4. Fm - Fluorescência máxima da clorofila $a\left(\right.$ (elétrons quantum ${ }^{-1}$ ) de plantas de mandioca em diferentes épocas após a aplicação de herbicidas.

\begin{tabular}{lcccc}
\hline Tratamentos & 2 DAA & 9 DAA & 16 DAA & 23 DAA \\
Bentazon & $412,00 \mathrm{c}$ & $235,00 \mathrm{c}$ & $215,00 \mathrm{c}$ & $215,50 \mathrm{a}$ \\
Clomazone & $823,25 \mathrm{a}$ & $683,75 \mathrm{a}$ & $978,25 \mathrm{a}$ & $384,25 \mathrm{a}$ \\
Fomesafen & $737,50 \mathrm{a}$ & $686,50 \mathrm{a}$ & $887,00 \mathrm{~b}$ & $337,50 \mathrm{a}$ \\
Fluazifop-buthyl & $731,00 \mathrm{a}$ & $697,25 \mathrm{a}$ & $816,75 \mathrm{~b}$ & $332,75 \mathrm{a}$ \\
Glyphosate & $546,50 \mathrm{~b}$ & $0,00 \mathrm{~d}$ & $0,00 \mathrm{~d}$ & $0,00 \mathrm{~b}$ \\
Nicosulfuron & $655,75 \mathrm{a}$ & $547,00 \mathrm{~b}$ & $773,00 \mathrm{~b}$ & $301,00 \mathrm{a}$ \\
Chlorimuron-ethyl & $711,50 \mathrm{a}$ & $593,25 \mathrm{~b}$ & $863,00 \mathrm{~b}$ & $304,75 \mathrm{a}$ \\
Fomesafen+Fluazifop & $511,75 \mathrm{~b}$ & $649,25 \mathrm{a}$ & $846,00 \mathrm{~b}$ & $399,25 \mathrm{a}$ \\
Sulfentrazone & $179,50 \mathrm{~d}$ & $0,00 \mathrm{~d}$ & $0,00 \mathrm{~d}$ & $0,00 \mathrm{~b}$ \\
Testemunha & $755,50 \mathrm{a}$ & $672,75 \mathrm{a}$ & $1031,00 \mathrm{a}$ & $321,25 \mathrm{a}$ \\
\hline CV $(\%)$ & \multicolumn{5}{c}{16,23} \\
\hline
\end{tabular}

Médias seguidas pela mesma letra na coluna não diferem entre si pelo teste de Scott Knott a 5\% de probabilidade. DAA: Dias após a aplicação.

Fonte: Elaboração dos autores.

Para a relação fluorescência variável e fluorescência máxima $(\mathrm{Fv} / \mathrm{Fm})$ das plantas de mandioca, observou-se aos 2 DAA que os herbicidas que proporcionaram os maiores valores da variável foram clomazone, fomesafen, fluazifopp-buthyl, chlorimuron-ethyl e nicosulfuron (Tabela 5). Considerando que plantas em perfeito estado fisiológico, na ausência de estresses, apresenta a relação $\mathrm{Fv} / \mathrm{Fm}$ variando de 0,75 a 0,85 , pode-se afirmar que o nicosulfuron, mesmo não diferindo da testemunha, promoveu algum tipo de estresse nas plantas de mandioca. Já o glyphosate, a mistura fluazifop-p-buthyl + fomesafen, bentazon e sulfentrazone causaram maior redução da variável. $\mathrm{O}$ rendimento quântico máximo do PS II $\left(\mathrm{F}_{\mathrm{v}} / \mathrm{F}_{\mathrm{m}}\right)$ pode variar de 0,75 a 0,85 em plantas não submetidas a estresses (BOLHÀR-NORDENKAMPF et al., 1989), sendo a redução desta razão um excelente indicador de efeito fotoinibidor quando as plantas estão submetidas ao estresse químico (ARAUS; HOGAN, 1994). Os valores inferiores a esses indicaram estresse e redução da eficiência quântica máxima do fotossistema II e, por consequência, do potencial fotossintético da planta. Importante destacar que apesar do bentazon ter apresentado níveis de intoxicação que variaram de 5 a $21 \%$ semelhantes (Tabela 2) ao observado para o chlorimuron-ethyl, deve-se enfocar que para o bentazon constatou-se durante todo o período de avaliação valores de Fv/Fm abaixo da faixa ideal e para o chlorimuron-ethyl esses valores estiveram dentro dessa faixa considerada ideal.

Os resultados da relação $\mathrm{Fv} / \mathrm{Fm}$ avaliados aos 9, 16 e 23 DAA mostram a formação de três grupos (Tabela 5). Plantas tratadas com a mistura fluazifop-p-buthyl + fomesafen que nos primeiros 2 DAA apresentavam baixa relação $\mathrm{Fv} / \mathrm{Fm}$, aos 9, 21, 23 DAA entraram na faixa ideal de valores dessa relação. No entanto, as plantas tratadas com bentazon mostraram baixo valor da relação Fv/Fm diferindo da testemunha. Já as plantas tratadas com o nicosulfuron apesar de não se diferenciarem da testemunha mostram valores da relação $\mathrm{Fv} / \mathrm{Fm}$ um pouco inferiores a faixa de 0,75 , indicando estresse provocado pelo herbicida no aparato fotossintético (Tabela 5). 
Tabela 5. Relação fluorescência variável e fluorescência máxima $(\mathrm{Fv} / \mathrm{Fm})$ da clorofila $a$ de plantas de mandioca em diferentes épocas após a aplicação de herbicidas.

\begin{tabular}{lcccc}
\hline Tratamentos & 2 DAA & 9 DAA & 16 DAA & 23 DAA \\
Bentazon & $0,37 \mathrm{c}$ & $0,17 \mathrm{~b}$ & $0,20 \mathrm{~b}$ & $0,38 \mathrm{~b}$ \\
Clomazone & $0,77 \mathrm{a}$ & $0,77 \mathrm{a}$ & $0,80 \mathrm{a}$ & $0,78 \mathrm{a}$ \\
Fomesafen & $0,82 \mathrm{a}$ & $0,78 \mathrm{a}$ & $0,82 \mathrm{a}$ & $0,81 \mathrm{a}$ \\
Fluazifop-buthyl & $0,81 \mathrm{a}$ & $0,79 \mathrm{a}$ & $0,82 \mathrm{a}$ & $0,82 \mathrm{a}$ \\
Glyphosate & $0,69 \mathrm{~b}$ & $0,00 \mathrm{c}$ & $0,00 \mathrm{c}$ & $0,00 \mathrm{c}$ \\
Nicosulfuron & $0,74 \mathrm{a}$ & $0,70 \mathrm{a}$ & $0,73 \mathrm{a}$ & $0,75 \mathrm{a}$ \\
Chlorimuron-ethyl & $0,81 \mathrm{a}$ & $0,76 \mathrm{a}$ & $0,79 \mathrm{a}$ & $0,76 \mathrm{a}$ \\
Fomesafen+Fluazifop & $0,59 \mathrm{~b}$ & $0,77 \mathrm{a}$ & $0,81 \mathrm{a}$ & $0,82 \mathrm{a}$ \\
Sulfentrazone & $0,26 \mathrm{c}$ & $0,00 \mathrm{c}$ & $0,00 \mathrm{c}$ & $0,00 \mathrm{c}$ \\
Testemunha & $0,78 \mathrm{a}$ & $0,79 \mathrm{a}$ & $0,81 \mathrm{a}$ & $0,79 \mathrm{a}$ \\
\hline CV $(\%)$ & & & \\
\hline
\end{tabular}

Médias seguidas pela mesma letra na coluna não diferem entre si pelo teste de Scott Knott a 5\% de probabilidade. DAA: Dias após a aplicação.

Fonte: Elaboração dos autores.

A recuperação de plantas de mandioca tratadas com a mistura dos herbicidas fluazifop-p-buthyl + fomesafen foi relatada em outros estudos (SILVA et al., 2011, 2012b). De acordo com esses autores, a intoxicação é causada principalmente pelo fomesafen e a recuperação da mandioca deve-se, principalmente, ao surgimento de novas folhas com ausência dos sintomas de intoxicação. No entanto, através das variáveis fisiológicas não foi possível confirmar que a intoxicação da mistura é promovida pelo fomesafen.

Para a taxa de transporte de elétrons (ETR) foi observado que aos 2 DAA os herbicidas bentazon e sulfentrazone promoveram drástica redução nos valores de ETR das plantas de mandioca. Para essa época os demais herbicidas não se diferenciaram da testemunha (Tabela 6). Como o parâmetro ETR determina a taxa de transporte de elétrons fotossintético no PSII, o uso do valor do ETR permite detectar o efeito da atuação do herbicida em nível de concentração de 0,5 micromoles $\mathrm{dm}^{-3}$, enquanto o método tradicional, que inclui a medição do parâmetro $\mathrm{Fv} / \mathrm{Fm}$, permite detectar apenas em um nível de concentração que é 100 vezes maior (KORRES; FROUD-WILLIAMS; MOSS, 2003; ABBASPOOR; TEICHER; STREIBIG, 2006), justificando o uso da metodologia empregada. No entanto, ao observar a os valores de ETR aos 9, 16 e 23 DAA constatou-se que o comportamento das plantas de mandioca tratadas com os herbicidas foi semelhante ao descrito para a relação Fv/Fm. Com o glyphosate e o sulfentrazone apresentando valores de ETR nulos e o bentazon reduzindo drasticamente a variável.

Os valores encontrados para as variáveis fisiológicas após a aplicação do glyphosate eram esperados, visto que esse herbicida é caracterizado como sistêmico, não seletivo e tem sua ação na inibição da síntese dos aminoácidos aromáticos, agindo na rota do chiquimato (SILVA; FERREIRA; FERREIRA, 2007). A morte das plantas tratadas com esse herbicida é lenta, sendo que só foi possível caracterizar a morte da mandioca aos 9 DAA. Segundo Silva et al. (2012a) recomendam-se o uso de herbicidas à base de glyphosate em aplicações dirigidas, evitando atingir as folhas da cultura, e quando a mandioca esteja com aproximadamente cinco meses e com 30 a $40 \mathrm{~cm}$ de haste em relação ao solo.

Com relação ao bentazon, essa redução rápida do ETR nas primeiras avaliações do experimento está relacionada com o fato do herbicida pertencer 
ao mecanismo de ação que inibe o transporte de elétrons no fotossistema II, justamente onde o fluorômetro detecta os valores de ETR. Em trabalho desenvolvido por Dayan, Trindade e Velini (2009), foi monitorado o ETR em plantas de Digitaria sanguinalis e Abutilon theophrasti, quando submetidas à aplicação de amicarbazone e atrazine. A taxa de transporte de elétrons para ambas as espécies foi completamente inibida oito horas após a aplicação dos herbicidas.

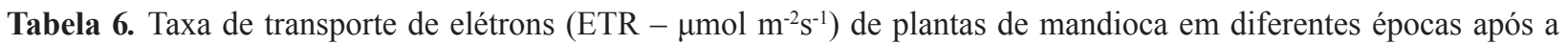
aplicação de herbicidas.

\begin{tabular}{|c|c|c|c|c|}
\hline Tratamentos & 2 DAA & 9 DAA & 16 DAA & 23 DAA \\
\hline Bentazon & $4,47 \mathrm{c}$ & $3,42 \mathrm{~b}$ & $5,62 b$ & $10,60 \mathrm{~b}$ \\
\hline Clomazone & $21,30 \mathrm{a}$ & $26,65 \mathrm{a}$ & $26,37 \mathrm{a}$ & $27,27 \mathrm{a}$ \\
\hline Fomesafen & $20,97 \mathrm{a}$ & $23,42 \mathrm{a}$ & $26,40 \mathrm{a}$ & $26,70 \mathrm{a}$ \\
\hline Fluazifop-buthyl & $20,90 \mathrm{a}$ & $24,05 \mathrm{a}$ & 26,76 a & $23,22 \mathrm{a}$ \\
\hline Glyphosate & $22,05 \mathrm{a}$ & $0,00 \mathrm{~b}$ & $0,00 \mathrm{~b}$ & $0,00 \mathrm{c}$ \\
\hline Nicossulfuron & $21,60 \mathrm{a}$ & $20,65 \mathrm{a}$ & $16,12 \mathrm{a}$ & $25,02 \mathrm{a}$ \\
\hline Chlorimuron-ethyl & $22,30 \mathrm{a}$ & $19,32 \mathrm{a}$ & $20,40 \mathrm{a}$ & $25,60 \mathrm{a}$ \\
\hline Fomesafen+Fluazifop & $16,27 \mathrm{a}$ & $24,40 \mathrm{a}$ & $26,97 \mathrm{a}$ & $26,57 \mathrm{a}$ \\
\hline Sulfentrazone & $12,05 \mathrm{~b}$ & $0,00 \mathrm{~b}$ & $0,00 \mathrm{~b}$ & $0,00 \mathrm{c}$ \\
\hline Testemunha & $22,07 \mathrm{a}$ & $25,00 \mathrm{a}$ & $25,05 \mathrm{a}$ & $26,22 \mathrm{a}$ \\
\hline $\mathrm{CV}(\%)$ & \multicolumn{4}{|c|}{21,96} \\
\hline
\end{tabular}

Médias seguidas pela mesma letra na coluna não diferem entre si pelo teste de Scott Knott a 5\% de probabilidade. DAA: Dias após a aplicação.

Fonte: Elaboração dos autores.

$\mathrm{Na}$ figura 1 encontra-se as curvas referentes a relação $\mathrm{Fv} / \mathrm{Fm}$, esta apresentado o comportamento de cada herbicidas nas quatro épocas de avaliação, sendo que, a linha pontilhada representa a faixa ideal da relação $\mathrm{Fv} / \mathrm{Fm}$, que varia de 0,75 a 0,85 , valores fora dessa faixa indicam algum estresse no aparato fotossintética das folhas das plantas de mandioca. Para o herbicida bentazon, a relação Fv/ Fm permaneceu fora da faixa ideal para todas as avaliações realizadas. Com relação ao glyphosate e ao sulfentrazone, constatou-se que na primeira época de avaliação as plantas apresentaram baixa relação $\mathrm{Fv} / \mathrm{Fm}$ com esses valores reduzindo para zero nas três últimas épocas de avaliação. A relação $\mathrm{Fv} / \mathrm{Fm}$ permaneceu na faixa ideal durante todos os períodos de avaliação para as plantas tratadas com os herbicidas clomazone, fomesafen, fluazifop-pbuthyl e chlorimuron-ehtyl.

O clomazone é um dos princípios ativos registradas para aplicação em pré-emergência da mandioca (BRASIL, 2013). A aplicação em pós-emergência do produto confirma a aparente seletividade dos herbicidas inibidores da síntese de carotenóides, conforme observados nos trabalhos de Silva et al. (2012b) e Silveira et al. (2012, 2013).

Aresposta da mandioca a aplicação dos herbicidas em pós-emergência variou com o produto aplicado. O glyphosate e sulfentrazone promoveram a morte das plantas. Os herbicidas clomazone, fomesafen, fluazifop-p-buthyl e clorimuron causaram baixa intoxicação nas plantas de mandioca e não alteraram a relação $\mathrm{Fv} / \mathrm{Fm}$ e a ETR. Para os herbicidas nicosulfuron e fluazifop-p-buthyl + fomesafen apesar dos valores de Fv/Fm estarem abaixo do ideal nas primeiras épocas de avaliação, as plantas se recuperaram nas ultimas épocas de avaliação. As variáveis fisiológicas avaliadas podem ser utilizadas como forma de avaliar a seletividade de herbicidas a mandioca, pois apresentaram comportamento semelhante aos observados para a intoxicação visual da cultura. 
Figura 1. Relação fluorescência variável e fluorescência máxima ( $\mathrm{Fv} / \mathrm{Fm})$ da clorofila $a$ de folhas de mandioca em diferentes épocas após a aplicação de herbicidas.
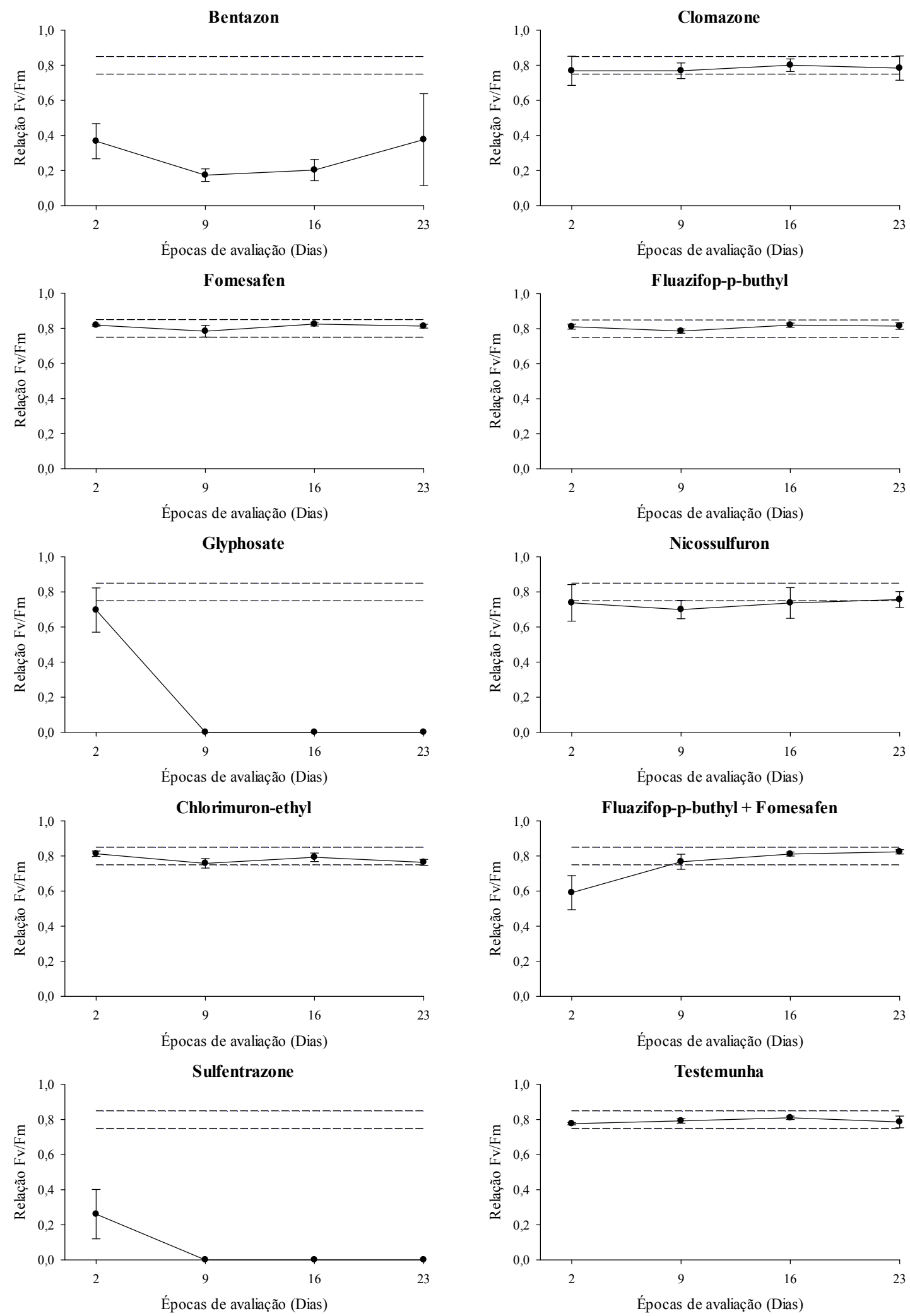

Fonte: Elaboração dos autores. 


\section{Agradecimentos}

Os autores agradecem ao Conselho Nacional de Desenvolvimento Científico e Tecnológico (CNPq), a Coordenação de Aperfeiçoamento de Pessoal de Nível Superior (CAPES) e Fundação de Amparo a Pesquisa do Estado de Minas Gerais (FAPEMIG) pelo apoio financeiro e as bolsas concedidas.

\section{Referências}

ABBASPOOR, M.; TEICHER, H. B.; STREIBIG, J. C. The effect of root-absorbed PSII inhibitors on Kautsky curve parameters in sugar beet. Weed Research, Washington, v. 46, n. 3, p. 226-235, 2006.

ARAUS, J. L.; HOGAN, K. P. Comparative leaf structure and patterns of photoinhibition of the neotropical palms. Scheelea zonensis and Socratea durissima growing in clearing and forest understory during the dry season in Panama. American Journal of Botanyv, St. Louis, v. 81, n. 6, p. 726-738, 1994.

BELKHODJA, R.; MORALES, F.; ABADIA, A.; GOMEZ-APARISI, J.; ABADIA, J. Chlorophyll fluorescence as a possible tool for salinity tolerance screening in barley (Hordeum vulgare L.). Plant Physiology, Glasgow, v. 104, n. 2, p. 667-673, 1994.

BOLHÀR-NORDENKAMPH, H. R.; LONG, R. S. P.; BAKER, N. R.; OQUIST, G.; SCHREIBER, U.; LECHNER, E. G. Chlorophyll fluorescence as a probe of the photossinthetic competence of leaves in the field: a review of current instrumentation. Functional Ecology, London, v. 3, n. 4, p. 497-514, 1989.

BOWN, A. W.; HALL, D. E.; MACGREGOR, K. B. Insect footsteps on leaves stimulate the accumulation of 4aminobutyrate and can be visualized through increased chlorophyll fluorescence and superoxide production. Plant Physiology, Glasgow, v. 129, n. 4, p. 1430-1434, 2002.

BRASIL. Ministério da Agricultura, Pecuária e Abastecimento. Coordenação-Geral de Agrotóxicos e Afins. Diário Oficial [da] União, Brasília, DF, 1 nov. 2013. Seção 1, p. 4. Disponível em: <http://extranet. agricultura.gov.br/agrofit_cons/principal_agrofit_cons.>. 2013. Acesso em: 1 jun. 2013.

CATUNDA, M. G.; FREITAS, S. P.; OLIVEIRA, J. G.; SILVA, C. M. M. Efeitos de herbicidas na atividade fotossintética e no crescimento de abacaxi (Ananas comossus). Planta daninha, Viçosa, v. 23, n. 1, p. 115121, 2005.
DAYAN, F. E.; TRINDADE, M. L. B.; VELINI, E. D. Amicarbazone, a new photosystem II inhibitor. Weed Science, Washington, v. 57, n. 6, p. 579-583, 2009.

FERRAZ, R. L. S.; BELTRÃO, N. E. M.; MELO, A. S.; MAGALHÃES, I. D.; FERNANDES, P. D.; ROCHA, M. $\mathrm{S}$. Trocas gasosas e eficiência fotoquímica de cultivares de algodoeiro herbáceo sob aplicação de silício foliar. Semina: Ciências Agrárias, Londrina, v. 35, n. 2, p. 735 748, 2014.

GALON, L.; FERREIRA, F. A.; FERREIRA, E. A.; SILVA, A. A.; SILVA, A. F.; ASPIAZÚ, I.; CONCENÇO, G.; FIALHO, C. M. T.; SANTOS, E. A.; TIRONI, S. P.; BARBOSA, M. H. P. Seletividade de herbicidas a genótipos de cana-de-açúcar. Planta Daninha, Viçosa, v. 27, p. 1083-1093, 2009. Especial.

GALON, L.; FERREIRA, F. A.; FERREIRA, E. A.; SILVA, A. A.; CONCENÇO, G.; SILVA, A. F.; REIS, M. R.; ASPIAZÚ, I.; FIALHO, C. M. T.; BARBOSA, M. H. P.; TIRONI, S. P. Tolerância de novos genótipos de canade-açúcar a herbicidas. Planta Daninha, Viçosa, v. 28, n. 2, p. 329-338, 2010.

GIROTTO, M.; ARALDI, R.; VELINI, E. D.; JASPER, S. P.; CARBONARI, C. A.; GOMES, G. L. G. C. Eficiência fotossintética da cana-de-açúcar após a aplicação dos herbicidas S-metolachlor e atrazine em pós-emergência. Revista Brasileira de Herbicidas, Maringá, v. 9, n. 3, p. 109-116, 2010.

IRELAND, C. R.; PERCIVAL, M. P.; BAKER, N. R. Modification of the induction of photosynthesis in wheat by glyphosate, an inhibitor of amino acid metabolism. Journal of Experimental Botany, Oxford, v. 37, n. 176, p. 299-308, 1986.

KORRES, N. E.; FROUD-WILLIAMS, R. J.; MOSS, S. R. Chlorophyll fluorescence technique as a rapid diagnostic test of the effects of the photosynthetic inhibitor chlortoluron on two winter wheat cultivars. Annals of Applied Biology, Malden, v. 143, n. 1, p. 5356, 2003.

KRAUSE, G. H.; WINTER, K. Photoinhibition of photosynthesis in plants growing in natural tropical forest gaps: a chlorophyll fluorescence study. Botanica Acta, Berlim, v. 109, n. 6, p. 456-462, 1996.

MAXWELL, K.; JOHNSON, G. N. Chlorophyll fluorescence: a practical guide. Journal of Experimental Botany, Oxford, v. 51, n. 345, p. 659-668, 2000.

MAZZA, C. A.; BOCCALANDRO, H. E.; GIORDANO, C. V.; BATTISTA, D.; SCOPEL, A. L.; BALLARE, C. L. Functional significance and induction by solar radiation 
of ultraviolet-absorbing sunscreens in field-grown soybean crops. Plant Physiology, Glasgow, v. 122, n. 1, p. 117-126, 2000.

NEGRISOLI, E.; VELINI, E. D.; TOFOLI, G. R.; CAVENAGHI, A. L.; MARTINS, D.; MORELLI, J. L.; COSTA, A. G. F. Seletividade de herbicidas aplicados em pré-emergência na cultura de cana-de-açúcar tratada com nematicidas. Planta Daninha, Viçosa, v. 22, n. 4, p. 567$575,2004$.

TORRES NETTO, A.; CAMPOSTRINI, E.; OLIVEIRA, G. J.; SMITH, R. E. B. Photosynthetic pigments, nitrogen, chlorophyll a fluorescence and SPAD-502 readings in coffee leaves. Scientia Horticulturae, Amsterdam, v. 104, n. 2, p. 199-209, 2005.

OLIVEIRA, J. G.; ALVES, P. L. C. A.; MAGALHÃES, A. C. The effect of chilling on the photosynthethic activith in coffe (Coffea arabica L.) seedlings. The protective action of chloroplastid pigments. Brazilian Journal of Plant Physiology, Campos dos Goytacazes, v. 14, n. 2, p. 95-104, 2002.

RODEN, J. S.; BALL, M. C. The effect of elevated $\left[\mathrm{CO}_{2}\right]$ on growth and photosynthesis of two eucalyptus species exposed to high temperatures and water deficits. Plant Physiology, Glasgow, v. 111, n. 3, p. 909-919, 1996.

SILVA, A. A.; FERREIRA, F. A.; FERREIRA, L. R. Herbicidas: classificação e mecanismo de ação. In: SILVA, A. A.; SILVA, J. F. Tópicos em manejo de plantas daninhas. Viçosa, MG: Editora UFV, 2007. p. 58-117.

SILVA, D. V.; SANTOS, J. B.; SILVEIRA, H. M.; CARVALHO, F. P.; CASTRO NETO, M. D.; FERREIRA, E. A.; SILVA, A. A.; CECON, P. R. Tolerância de cultivares de mandioca aos herbicidas fomesafen e fluazifop-p-butil. Revista Brasileira de Herbicidas, Maringá, v. 10, n. 3, p. 19-231, 2011.
SILVA, D. V.; SANTOS, J. B.; FERREIRA, E. A.; FRANÇA, A. C.; SILVA, A. A.; SEDIYAMA, T. Manejo de plantas daninhas na cultura da mandioca. Planta daninha, Viçosa, v. 30, n. 4, p. 901-910, 2012a.

SILVA, D. V.; SANTOS, J. B.; CARVALHO, F. P.; FERREIRA, E. A.; FRANÇA, A. C.; FERNANDES, J. S. C.; GANDINI, E. M. M.; CUNHA, V. C. Seletividade de herbicidas pós-emergentes na cultura da mandioca. Planta Daninha, Viçosa, v. 30, n. 4, p. 835-841, 2012 b.

SILVEIRA, H. M.; SILVA, D. V.; SANTOS, J. B.; CASTRONETO, M.D.; FERREIRA,A.F.; CARVALHO, F. P.; SILVA, A. A.; SEDIYAMA, T. Sensibilidade de cultivares de mandioca ao herbicida mesotrione. Revista Brasileira de Herbicidas, Maringá, v. 11, n. 1, p. 24-31, 2012.

SILVEIRA, H. M; FERREIRA, E. A.; SILVA, D. V.; CASTRO NETO, M. D. C.; CARVALHO, F. P.; SANTOS, J. B.; SILVA, A. A. Características fisiológicas de cultivares de mandioca após aplicação do mesotrione. Planta daninha, Viçosa, v. 31, n. 2, p. 403-409, 2013.

TORRES, L. G.; FERREIRA, E. A.; ROCHA, P. R. R.; FARIA, A. T.; GONÇALVES, V. A.; GALON, L.; SILVA, A. F.; SILVA, A. A. Alterações nas características fisiológicas de cultivares de cana-de-açúcar submetida à aplicação de herbicidas. Planta Daninha, Viçosa, v. 30, n. 3 , p. $581-587,2012$.

YOUNG, A. L.; FRANK, H. A. Energy transfer reactions involving carotenoids: quenching of chlorophyll fluorescence. Journal of Photochemistry and Photobiology B: Biology, New York, v. 36, n. 1, p. 3-15, 1996. 
